\title{
HARDY OPERATORS IN THE LOCAL "COMPLEMENTARY" GENERALIZED VARIABLE EXPONENT WEIGHTED MORREY SPACES
}

\author{
Zuleyxa O. Azizova \\ Azerbaijan State Oil and Industry University \\ AZ 1010, Baku, 20 Azadlig Ave., AZERBAIJAN
}

\begin{abstract}
In this paper we consider local "complementary" generalized weighted Morrey spaces ${ }^{c} \mathcal{M}_{\left\{x_{0}\right\}}^{p(\cdot), \varphi}(\Omega)$ with variable exponent $p(x)$ and a general function $\omega(r)$ defining the weighted Morrey-type norm. We prove the boundedness of the Hardy operators in the spaces ${ }^{c} \mathcal{M}_{\left\{x_{0}\right\}}^{p(\cdot), \omega}(\Omega)$ in case of unbounded sets $\Omega \subset R^{n}$.
\end{abstract}

AMS Subject Classification: 42B20, 42B25, 42B35

Key Words: local "complementary" generalized variable exponent weighted Morrey spaces; weighted Hardy operator

\section{Introduction}

Nowadays there is an evident increase of investigations, last two decades related to both the theory of variable exponent function spaces and operator theory in these spaces. We refer for instance to the surveying papers [3], [4], [24], on the progress in this field, including topics of Harmonic Analysis and Operator Theory, see also references therein. Variable exponent Morrey spaces $\mathcal{L}^{p, \lambda}\left(R^{n}\right)$, were introduced and studied in [1] in the Euclidean setting. In [1] the boundedness of the maximal operator was proved in variable exponent Morrey spaces $\mathcal{L}^{p, \lambda}\left(R^{n}\right)$ under the $\log$-condition on $p(\cdot)$ and $\lambda(\cdot)$, and for potential operators a Sobolev type $\mathcal{L}^{p(\cdot), \lambda(\cdot)} \rightarrow \mathcal{L}^{q(\cdot), \lambda(\cdot)}$-theorem was proved under the same log-condition in the case of bounded sets.

Received: April 27, 2020

(C) 2020 Academic Publications 
The generalized variable exponent Morrey spaces were introduced and studied in [11] in the case of bounded sets. In [11] the boundedness of the maximal operator, potential operators and singular integral operators in variable exponent Morrey spaces under the certain conditions were proved.

We consider the following Hardy operators

$$
\begin{gathered}
A_{\beta}^{\alpha} f(x)=|x|^{\alpha-n+\beta} \int_{|y| \leq|x|} \frac{f(y)}{|y|^{\beta}} d y, \\
\mathcal{A}_{\beta}^{\alpha} f(x)=|x|^{\alpha+\beta} \int_{|y|>|x|} \frac{f(y) d y}{|y|^{n+\beta}},
\end{gathered}
$$

where $\alpha \geq 0$.

We use the following notation: $R^{n}$ is the $n$-dimensional Euclidean space, $\Omega \subset R^{n}$ is an open set, $\chi_{E}(x)$ is the characteristic function of a set $E \subseteq R^{n}$, $\left.B(x, r)=\left\{y \in R^{n}:|x-y|<r\right\}\right), \widetilde{B}(x, r)=B(x, r) \cap \Omega$, by $c, C, c_{1}, c_{2}$ etc., we denote various absolute positive constants, which may have different values even in the same line.

\section{Variable exponent local "complementary" generalized Morrey spaces}

We refer to the book [5] for variable exponent Lebesgue spaces but give some basic definitions and facts. Let $p(\cdot)$ be a measurable function on $\Omega$ with values in $[1, \infty)$. An open set $\Omega$ is assumed to be bounded throughout the whole paper. We mainly suppose that

$$
1<p_{-} \leq p(x) \leq p_{+}<\infty,
$$

where $p_{-}:=\underset{x \in \Omega}{\operatorname{ess} \inf _{0}} p(x)>1, \quad p_{+}:=\underset{x \in \Omega}{\operatorname{ess} \sup } p(x)<\infty$. By $L^{p(\cdot)}(\Omega)$ we denote the space of all measurable functions $f(x)$ on $\Omega$ such that

$$
I_{p(\cdot)}(f)=\int_{\Omega}|f(x)|^{p(x)} d x<\infty .
$$

Equipped with the norm

$$
\|f\|_{p(\cdot)}=\inf \left\{\eta>0: I_{p(\cdot)}\left(\frac{f}{\eta}\right) \leq 1\right\}
$$

this is a Banach function space. By $p^{\prime}(\cdot)=\frac{p(x)}{p(x)-1}, x \in \Omega$, we denote the conjugate exponent. 
For the basics on variable exponent Lebesgue spaces we refer to [26], [16]. $\mathcal{P}(\Omega)$ is the set of bounded measurable functions $p: \Omega \rightarrow[1, \infty)$; $\mathcal{P}^{l o g}(\Omega)$ is the set of exponents $p \in \mathcal{P}(\Omega)$ satisfying the local log-condition

$$
|p(x)-p(y)| \leq \frac{A}{-\ln |x-y|}, \quad|x-y| \leq \frac{1}{2} \quad x, y \in \Omega,
$$

where $A=A(p)>0$ does not depend on $x, y$.

We will use also the following decay conditions:

$$
\begin{aligned}
& |p(x)-p(0)| \leq \frac{A_{0}}{|\ln | x||}, \quad|x| \leq \frac{1}{2}, \\
& |p(x)-p(\infty)| \leq \frac{A_{\infty}}{|\ln | x||}, \quad|x| \geq 2,
\end{aligned}
$$

where $p_{\infty}=\lim _{x \rightarrow \infty} p(x)>1$. $(2.2)$;

$\mathcal{A}^{\log }(\Omega)$ is the set of bounded exponents $p: \Omega \rightarrow R$ satisfying the condition

t $P^{\log }(\Omega)$ is the set of exponents $p \in \mathcal{P}^{\log }(\Omega)$ with $1<p_{-} \leq p(x) \leq p_{+}<\infty$;

for $\Omega$ which may be unbounded, by $\mathcal{P}_{\infty}(\Omega), \mathcal{P}_{\infty}^{\log }(\Omega), P_{\infty}^{\log }(\Omega), \mathcal{A}_{\infty}^{\log }(\Omega)$ we denote the subsets of the above sets of exponents satisfying the decay condition (2.4) (when $\Omega$ is unbounded).

For brevity, by $P_{0, \infty}^{\log }(\Omega)$ we denote the set of bounded measurable functions (not necessarily with values in $[1, \infty)$ ), which satisfy the decay conditions $(2.3)$ and (2.4).

By $\varphi$ we always denote a weight, i.e. a positive, locally integrable function with $R^{n}$. The weighted Lebesgue space $L_{\varphi}^{p(\cdot)}\left(R^{n}\right)$ is defined as the set of all measurable functions for which

$$
\|f\|_{L_{\varphi}^{p(\cdot)}\left(R^{n}\right)}=\|f \varphi\|_{L^{p(\cdot)}\left(R^{n}\right)} \cdot
$$

Let us define the class $A_{p(\cdot)}\left(R^{n}\right)$ (see [6]) to consist of those weights $\varphi$ for which

$$
[\varphi]_{A_{p(\cdot)}} \equiv \sup _{B}|B|^{-1}\|\varphi\|_{L^{p(\cdot)}(\widetilde{B}(x, r))}\left\|\varphi^{-1}\right\|_{L^{p^{\prime}(\cdot)}(\widetilde{B}(x, r))}<\infty .
$$

A weight function $\varphi$ belongs to the class $A_{p(\cdot), q(\cdot)}\left(R^{n}\right)$ if

$$
[\varphi]_{A_{p(\cdot), q(\cdot)}} \equiv \sup _{B} r^{\theta_{p}(x, r)-\theta_{q}(x, r)-n}\|\varphi\|_{L^{q(\cdot)}(\widetilde{B}(x, r))}\left\|\varphi^{-1}\right\|_{L^{p^{\prime}(\cdot)}(\widetilde{B}(x, r))}<\infty .
$$

Everywhere in the sequel the functions $\omega(r), \omega_{1}(r)$ and $\omega_{2}(r)$ used in the body of the paper, are non-negative measurable function on $(0, \infty)$. 
Definition 2.1. Let $x_{0} \in \Omega, 1 \leq p_{-} \leq p(x) \leq p_{+}<\infty$. The local "complementary" generalized Morrey space ${ }^{c} \mathcal{M}_{\left\{x_{0}\right\}}^{p(\cdot), \omega}(\Omega)$ is defined by the norms

$$
\begin{gathered}
\|f\|_{c \mathcal{M}_{\left\{x_{0}\right\}}^{p(\cdot), \omega}}=\sup _{r>0} \frac{r^{\theta_{p^{\prime}}\left(x_{0}, r\right)}}{\omega(r)}\|f\|_{L^{p(\cdot)}\left(\Omega \backslash \widetilde{B}\left(x_{0}, r\right)\right)}, \\
\|f\|_{c \mathcal{M}_{\left\{x_{0}\right\}}^{p(\cdot), \omega, \varphi}}=\sup _{r>0} \frac{\|\varphi\|_{L^{p^{\prime}(\cdot)}\left(\widetilde{B}\left(x_{0}, r\right)\right)}\|f\|_{L_{\varphi}^{p(\cdot)}\left(\Omega \backslash \widetilde{B}\left(x_{0}, r\right)\right)}}{\omega(r)} .
\end{gathered}
$$

Everywhere in the sequel we assume that

$$
\sup _{r>0} \frac{\|\varphi\|_{L^{p^{\prime}(\cdot)}\left(\widetilde{B}\left(x_{0}, r\right)\right)}}{\omega(r)}<\infty,
$$

which makes the space ${ }^{c} \mathcal{M}_{\left\{x_{0}\right\}}^{p(\cdot), \omega}(\Omega)$ non-trivial, since it contains $L^{p(\cdot)}(\Omega)$ in this case.

If also $\inf _{r>0} \frac{1}{\omega(r)}>0$, then ${ }^{c} \mathcal{M}_{\left\{x_{0}\right\}}^{p(\cdot), \omega}(\Omega)=L^{p(\cdot)}(\Omega)$. Therefore, to guarantee that the "complementary" space ${ }^{c} \mathcal{M}_{\left\{x_{0}\right\}}^{p(\cdot), \omega}(\Omega)$ is strictly larger than $L^{p}(\Omega)$, one should be interested in the cases where

$$
\lim _{r \rightarrow 0} \frac{r^{\theta_{p^{\prime}}\left(x_{0}, r\right)}}{\omega(r)}=0 .
$$

Clearly, the space ${ }^{c} \mathcal{M}_{\left\{x_{0}\right\}}^{p(\cdot), \omega}(\Omega)$ may contain functions with a non-integrable singularity at the point $x_{0}$, if no additional assumptions are introduced.

\section{Weighted Hardy operator in the spaces ${ }^{c} \mathcal{M}_{\{0\}}^{p(\cdot), \omega, \varphi}\left(R^{n}\right)$}

The proof of the main result of this section presented in Theorems 3.1 and 3.2 is based on the estimate given in the following preliminary theorems.

Theorem 3.1. Let $p \in P_{0, \infty}^{\log }\left(R^{n}\right), \varphi \in A_{p(\cdot), q(\cdot)}\left(R^{n}\right)$. Suppose also that $\beta \geq 0$ and the functions $\left(\omega_{1}, \omega_{2}\right)$ satisfy the conditions

$$
\begin{gathered}
\int_{0}^{t} \frac{s^{\alpha-\theta_{p}(0, s)+\theta_{q}(0, s)} \omega_{1}(s)}{\|\varphi\|_{L^{p^{\prime}(\cdot)}(B(0, s))}} \frac{d s}{s} \leq C \frac{\omega_{2}(t)}{\|\varphi\|_{L^{q^{\prime}(\cdot)}(B(0, s))}} \\
\int_{0}^{t} \frac{\omega_{1}(s)}{s^{\beta}\|\varphi\|_{L^{p^{\prime}(\cdot)}(B(0, s))}} \frac{d s}{s} \leq C \frac{\omega_{1}(t)}{t^{\beta}\|\varphi\|_{L^{p^{\prime}(\cdot)}(B(0, t))}}
\end{gathered}
$$

where $t>0$. 
Then the weighted Hardy operator $A_{\beta}^{\alpha}$ is bounded from the space ${ }^{c} \mathcal{M}_{\{0\}}^{p(\cdot), \omega_{1}, \varphi}\left(R^{n}\right)$ to the space ${ }^{c} \mathcal{M}_{\{0\}}^{q(\cdot), \omega_{2}, \varphi}\left(R^{n}\right)$.

Proof. Let $f \in{ }^{c} \mathcal{M}_{\{0\}}^{p(\cdot), \omega_{1}, \varphi}\left(R^{n}\right), \delta>0$. We have

$$
\begin{aligned}
\int_{|z|<r} \frac{|f(z)|}{|z|^{\beta}} d z & =\int_{B(0, r)} \frac{|f(z)|}{|z|^{\beta}} d z=\delta \int_{B(0, r)} \frac{|f(z)|}{|z|^{\beta+\delta}}\left(\int_{0}^{|z|} s^{\delta-1} d s\right) d z \\
& =\delta \int_{0}^{r} s^{\delta-1}\left(\int_{\left\{z \in R^{n}: s<|z|<r\right\}} \frac{|f(z)|}{|z|^{\beta+\delta}} d z\right) d s .
\end{aligned}
$$

Applying Hölder inequality, we get

$$
\int_{|z|<r} \frac{|f(z)|}{|z|^{\beta}} d z \leq C \int_{0}^{r} \frac{1}{s^{\beta}}\|f\|_{L^{p(\cdot), \varphi\left(R^{n} \backslash B(0, s)\right)} \|}\left\|\varphi^{-1}\right\|_{L^{p^{\prime}(\cdot)}(B(0, r))} \frac{d s}{s} .
$$

Then we have

$$
\begin{gathered}
\int_{|z|<r} \frac{|f(z)|}{|z|^{\beta}} d z \\
\leq C\left\|\varphi^{-1}\right\|_{L^{p^{\prime}(\cdot)}(B(0, r))} \int_{0}^{r} \frac{1}{s^{\beta}}\|f\|_{L^{p(\cdot)}\left(R^{n} \backslash B(0, s)\right)} \frac{d s}{s} .
\end{gathered}
$$

Therefore by (3.3), (3.2) and (3.1) we have

$$
\begin{aligned}
& \left\|A_{\beta}^{\alpha} f\right\|_{L^{q(\cdot), \varphi}\left(R^{n} \backslash B(0, t)\right)} \\
& \leq C\|\| \varphi^{-1} \|_{L^{p^{\prime}(\cdot)(B(0,|\cdot|))}}|\cdot|^{\alpha-n+\beta} \\
& \times \int_{0}^{|\cdot|} \frac{\|f\|_{L^{p(\cdot), \varphi}\left(R^{n} \backslash B(0, s)\right)}}{s^{\beta}} \frac{d s}{s} \|_{L^{q(\cdot), \varphi}\left(R^{n} \backslash B(0, t)\right)} \\
& \leq C\|f\|_{c \mathcal{M}_{\{0\}}^{p(\cdot), \omega_{1}, \varphi}\left(R^{n}\right)} \\
& \times\left\|\frac{|\cdot|^{\alpha-\theta_{p}(0,|\cdot|)+\theta_{q}(0,|\cdot|)+\beta}}{\|\varphi\|_{L^{q(\cdot)}(B(0,|\cdot|))}} \int_{0}^{|\cdot|} \frac{\omega_{1}(s)}{s^{\beta}\|\varphi\|_{L^{p^{\prime}(\cdot)}(B(0, s))}} \frac{d s}{s}\right\|_{L^{q(\cdot), \varphi}\left(R^{n} \backslash B(0, t)\right)} \\
& \leq C\|f\|_{c \mathcal{M}_{\{0\}}^{p(\cdot), \omega_{1}, \varphi}\left(R^{n}\right)}
\end{aligned}
$$




$$
\begin{gathered}
\times\left\|\frac{|\cdot|^{\alpha-\theta_{p}(0,|\cdot|)+\theta_{q}(0,|\cdot|)+\beta} \omega_{1}(|\cdot|)}{|\cdot| \beta\|\varphi\|_{L^{p^{\prime}(\cdot)}(B(0,|\cdot|))}\|\varphi\|_{L^{q(\cdot)}(B(0,|\cdot|))}}\right\|_{L^{q(\cdot), \varphi}\left(R^{n} \backslash B(0, t)\right)} \\
\leq C\|f\|_{\mathcal{M}^{\prime} \mathcal{M}_{\{0\}}^{p(\cdot), \omega_{1}, \varphi}\left(R^{n}\right)} \\
\times\left\|\frac{|\cdot|^{\alpha-\theta_{p}(0,|\cdot|)+\theta_{q}(0,|\cdot|)} \omega_{1}(|\cdot|)}{\|\varphi\|_{L^{q(\cdot)}(B(0,|\cdot|))}\|\varphi\|_{L^{p^{\prime}(\cdot)}(B(0,|\cdot|))}}\right\|_{L^{q(\cdot), \varphi}\left(R^{n} \backslash B(0, t)\right)} \\
\leq C\|f\|_{\mathcal{M}_{\{0\}}^{p(\cdot), \omega_{1}, \varphi}\left(R^{n}\right)} \int_{0}^{t} \frac{s^{\alpha-\theta_{p}(0, s)+\theta_{q}(0, s)} \omega_{1}(s)}{\|\varphi\|_{L^{p^{\prime}(\cdot)}(B(0, s))}} \frac{d s}{s} \\
\leq C\|f\|_{c \mathcal{M}_{\{0\}}^{p(\cdot), \omega_{1}, \varphi}\left(R^{n}\right)} \frac{\omega_{2}(t)}{\|\varphi\|_{L^{q^{\prime}(\cdot)}(B(0, s))}}
\end{gathered}
$$

Theorem 3.2. Let $p \in P_{0, \infty}^{\log }\left(R^{n}\right), \varphi \in A_{p(\cdot), q(\cdot)}\left(R^{n}\right)$ Suppose also that $\beta<-n$ and the functions $\left(\omega_{1}, \omega_{2}\right)$ satisfy the conditions

$$
\begin{gathered}
\int_{0}^{t} \frac{s^{\alpha-\theta_{p}(0, s)+\theta_{q}(0, s)} \omega_{1}(s)}{\|\varphi\|_{L^{p^{\prime}(\cdot)}(B(0, s))}} \frac{d s}{s} \leq C \frac{\omega_{2}(t)}{\|\varphi\|_{L^{q^{\prime}(\cdot)}(B(0, t))}} \\
\int_{t}^{\infty} \frac{s^{-\theta_{p}(0, s)+\theta_{q}(0, s)-\beta-1}}{\|\varphi\|_{L^{q(\cdot)}(B(0, s))}} d s \leq C \frac{t^{-\theta_{p}(0, t)+\theta_{q}(0, t)-\beta}}{\|\varphi\|_{L^{q(\cdot)}(B(0, t))}}
\end{gathered}
$$

where $t>0$.

Then the weighted Hardy operator $\mathcal{A}_{\beta}^{\alpha}$ is bounded from the space ${ }^{c} \mathcal{M}_{\{0\}}^{p(\cdot), \omega_{1}, \varphi}\left(R^{n}\right)$ to the space ${ }^{c} \mathcal{M}_{\{0\}}^{q(\cdot), \omega_{2}, \varphi}\left(R^{n}\right)$.

Proof. Let $f \in \mathcal{M}_{\{0\}}^{p(\cdot), \omega_{1}, \varphi}\left(R^{n}\right), \delta>0,-\delta<n+\beta$. We have

$$
\begin{aligned}
\int_{|z|>r} \frac{|f(z)|}{|z|^{n+\beta}} d z & =\int_{R^{n} \backslash B(0, r)} \frac{|f(z)|}{|z|^{n+\beta}} d z \\
& =\delta \int_{R^{n} \backslash B(0, r)}|z|^{-n-\beta-\delta}|f(z)|\left(\int_{0}^{|z|} s^{\delta-1} d s\right) d z \\
& =\delta \int_{r}^{\infty} s^{\delta-1}\left(\int_{\left\{z \in R^{n}: r<|z|<s\right\}}|z|^{-n-\beta-\delta}|f(z)| d z\right) d s
\end{aligned}
$$


Hence applying Hölder inequality we get

$$
\begin{gathered}
\int_{|z|>r} \frac{|f(z)|}{|z|^{n+\beta}} d z \\
\leq C \int_{r}^{\infty} s^{-n-\beta-1}\|f\|_{L_{\varphi}^{p(\cdot)}\left(R^{n} \backslash B(0, r)\right)}\left\|\varphi^{-1}\right\|_{L^{p^{\prime}(\cdot)(B(0, s))}} d s .
\end{gathered}
$$

Therefore by (3.6), we obtain

$$
\begin{aligned}
& \left\|\mathcal{A}_{\beta}^{\alpha} f\right\|_{L_{\varphi}^{q(\cdot)}\left(R^{n} \backslash B(0, t)\right)} \\
& \leq C\left\||\cdot|^{\alpha+\beta}\right\| f \|_{L_{\varphi}^{p(\cdot)}\left(R^{n} \backslash B(0,|\cdot|)\right)} \\
& \times \int_{|\cdot|}^{\infty} s^{-n-\beta-1}\left\|\varphi^{-1}\right\|_{L^{p^{\prime}(\cdot)}(B(0, s))} d s \|_{L_{\varphi}^{q(\cdot)}\left(R^{n} \backslash B(0, t)\right)} \\
& \leq C\left\||\cdot|^{\alpha+\beta}\right\| f\left\|_{L_{\varphi}^{p(\cdot)}\left(R^{n} \backslash B(0,|\cdot|)\right)} \int_{|\cdot|}^{\infty} \frac{s^{-\theta_{p}(0, s)+\theta_{q}(0, s)-\beta-1}}{\|\varphi\|_{L^{q(\cdot)}(B(0, s))}} d s\right\|_{L_{\varphi}^{q(\cdot)}\left(R^{n} \backslash B(0, t)\right)} \\
& \leq C\|f\|_{c \mathcal{M}_{\{0\}}^{p(\cdot), \omega_{1}, \varphi}\left(R^{n}\right)} \\
& \times\left\|\frac{|\cdot|^{\alpha+\beta} \omega_{1}(|\cdot|)}{\|\varphi\|_{L^{p^{\prime}(\cdot)}(B(0,|\cdot|))}} \int_{|\cdot|}^{\infty} \frac{s^{-\theta_{p}(0, s)+\theta_{q}(0, s)-\beta-1}}{\|\varphi\|_{L^{q(\cdot)}(B(0, s))}} d s\right\|_{L_{\varphi}^{q(\cdot)}\left(R^{n} \backslash B(0, t)\right)} \\
& \leq C\|f\|_{\mathcal{M}_{\{0\}}^{p(\cdot), \omega_{1}, \varphi}\left(R^{n}\right)}\left\|\frac{|\cdot|^{\alpha-\theta_{p}(0,|\cdot|)+\theta_{q}(0,|\cdot|)} \omega_{1}(|\cdot|)}{\|\varphi\|_{L^{q(\cdot)}(B(0,|\cdot|))}\|\varphi\|_{L^{p^{\prime}(\cdot)}(B(0,|\cdot|))}}\right\|_{L_{\varphi}^{q(\cdot)}\left(R^{n} \backslash B(0, t)\right)} \\
& \leq C\|f\|_{c_{\mathcal{M}}^{p(\cdot), \omega_{1}, \varphi}\left(R^{n}\right)} \int_{0}^{t} \frac{s^{\alpha-\theta_{p}(0, s)+\theta_{q}(0, s)} \omega_{1}(s)}{\|\varphi\|_{L^{p^{\prime}(\cdot)}(B(0, s))}} \frac{d s}{s} \\
& \leq C\|f\|_{c \mathcal{M}_{\{0\}}^{p(\cdot), \omega_{1}, \varphi}\left(R^{n}\right)} \frac{\omega_{2}(t)}{\|\varphi\|_{L^{q^{\prime}(\cdot)}(B(0, t))}} .
\end{aligned}
$$




\section{References}

[1] A. Almeida, J.J. Hasanov, S.G. Samko, Maximal and potential operators in variable exponent Morrey spaces, Georgian Math. J., 15, No 2 (2008), $1-15$.

[2] Z.O. Azizova, J.J. Hasanov, The weighted Hardy operator and it is commutator on Orlicz-Morrey spaces, Intern. J. of Pure and Appl. Math., 118, No 2 (2018), 385-395.

[3] D. Cruz-Uribe, A. Fiorenza, J.M. Martell, C. Perez, The boundedness of classical operators on variable $L^{p}$ spaces, Ann. Acad. Scient. Fennicae, Math., 31 (2006), 239-264.

[4] L. Diening, Maximal functions on generalized Lebesgue spaces $L^{p(x)}$, Math. Inequal. Appl., 7, No 2 (2004), 245-253.

[5] L. Diening, P. Harjulehto, P. Hästö, M. Ružička, Lebesgue and Sobolev Spaces with Variable Exponents, Lecture Notes in Mathematics, SpringerVerlag, Berlin (2011).

[6] L. Diening, P. Hästö, A. Nekvinda, Open problems in variable exponent Lebesgue and Sobolev spaces, In: 'Function Spaces, Differential Operators and Nonlinear Analysis', Proc. of the Conference held in Milovy, Bohemian-Moravian Uplands, May 28 - June 2, 2004, Math. Inst. Acad. Sci. Czech Republick, Praha (2005), 38-58.

[7] L. Diening, M. Rüźićka, Calderón-Zygmund operators on generalized Lebesgue spaces $L^{p(\cdot)}$ and problems related to fluid dynamics, J. Reine Angew. Math., 563 (2003), 197-220.

[8] G. Di Fazio, M.A. Ragusa, Commutators and Morrey spaces, Bollettino U.M.I., 7, No 5-A (1991), 323- 332.

[9] J. Garcia-Cuerva, E. Harboure, C. Segovia, J.L. Torrea, Weighted norm inequalities for commutators of strongly singular integrals, Indiana Univ. Math. J., 40, No 4 (1991), 1397-1420.

[10] V.S. Guliyev, S.G. Samko, Maximal, potential and singular operators in the generalized variable exponent Morrey spaces on unbounded sets, $J$. Math. Sci. (N. Y.), 193, No 2 (2013), 228-248. 
[11] V.S. Guliyev, J.J. Hasanov, S.G. Samko, Boundedness of the maximal, potential and singular operators in the generalized variable exponent Morrey spaces, Math. Scand., 107 (2010), 285-304.

[12] V.S. Guliyev, J.J. Hasanov, S.G. Samko, Boundedness of the maximal, potential and singular integral operators in the generalized variable exponent Morrey type spaces, J. Math. Sci., 170, No 4 (2010), 423-443.

[13] V.S. Guliyev, J.J. Hasanov, S.G. Samko, Maximal, potential and singular operators in the local "complementary" variable exponent Morrey type spaces, J. Math. Sci., 193, No 2 (2013), 228-248.

[14] A. Karlovich, A. Lerner, Commutators of singular integrals on generalized $L^{p}$ spaces with variable exponent, Publ. Math., 49, No 1 (2005), 111-125.

[15] V. Kokilashvili, A. Meskhi, Boundedness of maximal and singular operators in Morrey spaces with variable exponent, Arm. J. Math. (Electronic), 1, No 1 (2008), 18-28.

[16] O. Kovacik, J. Rakosnik, On spaces $L^{p(x)}$ and $W^{k, p(x)}$, Czechoslovak Math. J., 41, No 116 (1991), 592-618.

[17] C.B. Morrey, On the solutions of quasi-linear elliptic partial differential equations, Trans. Amer. Math. Soc., 43 (1938), 126-166.

[18] T. Mizuhara, Boundedness of some classical operators on generalized Morrey spaces, Harmonic Analysis (S. Igari, Ed.), ICM 90 Satellite Proceedings, Springer - Verlag, Tokyo (1991), 183-189.

[19] Y. Mizuta, T. Shimomura, Sobolev embeddings for Riesz potentials of functions in Morrey spaces of variable exponent, J. Math. Soc. Japan, 60 (2008), 583-602.

[20] D. Lukkassen, L.-E. Persson, S. Samko, P. Wall, Weighted Hardy operators in complementary Morrey Spaces, J. Funct. Spaces Appl., 2012; doi: $10.1155 / 2012 / 283285$.

[21] D. Lukkassen, L.-E.Persson, S. Samko, P.Wall, Weighted Hardy-type inequalities in variable exponent Morrey-type spaces, J. Funct. Spaces Appl., 2013; doi:10.1155/2013/716029.

[22] D. Lukkassen, L.-E.Persson, N. Samko, Hardy type operators in local vanishing Morrey spaces on fractal sets, Fract. Calc. Appl. Anal., 18, No 5 (2015), 1252-1276. 
[23] J. Peetre, On the theory of $\mathcal{L}_{p, \lambda}$ spaces, J. Funct. Anal., 4 (1969), 71-87.

[24] S. Samko, On a progress in the theory of Lebesgue spaces with variable exponent: maximal and singular operators, Integral Transforms Spec. Funct., 16, No 5-6 (2005), 461-482.

[25] C. Segovia, J.L. Torrea, Higher order commutators for vector-valued Calderón - Zygmund operators, Trans. Amer. Math. Soc., 336, No 2 (1993), 537-556.

[26] I.I. Sharapudinov, The topology of the space $\mathcal{L}^{p(t)}([0,1])$, Mat. Zametki, 26, No 3-4 (1979), 613-632. 